\title{
Multidisciplinary Design Optimization Of Aerospace Systems
}

Kamran Behdinan

Department of Aerospace Engineering, Ryerson University, Toronto, ON, Canada, M5B $2 \mathrm{~K} 3$ kbehdina@ryerson.ca

\begin{abstract}
This paper presents the evaluation of different MDO methodologies which take into consideration optimization and other factors influencing their structures. Illustrative comparisons are made for analytic functions and a supersonic business jet design case study. Results show the promising features of the proposed evaluation metrics to define a standardized guideline when dealing with multidisciplinary optimization formulations which can be applied to aerospace systems design problems.
\end{abstract}

\section{Introduction}

Multidisciplinary design optimization (MDO) is a methodology used in design of systems where there is the interaction of many different disciplines present. For example, Sobieski $\left.{ }^{1,2}\right]$ has given examples of aircraft optimization where structural, aerodynamic, and control disciplines are optimized at the same time. This method of optimization helps to answer the question presented by the American Institute of Aeronautics and Astronautics $\left[{ }^{2}\right]$ "How to decide what to change, and to what extent to change it, when everything influences everything else". Integrating many disciplines together may help to answer this question for designers.

In order to address the computational challenges that arise in MDO, different strategies have been proposed by defining a proper problem formulation or finding efficient optimization algorithms $\left[{ }^{1}, 2\right]$. Examples of these architectures include the following methods: Multi-Disciplinary Feasible (MDF) $\left[^{3}\right]$, Individual Discipline Feasible (IDF) $\left[{ }^{4}\right]$, Collaborative Optimization (CO) $\left.{ }^{5}\right]$, Concurrent Subspace Optimization (CSSO) $\left.{ }^{6}{ }^{6}\right]$, and Bi-Level Integrated Synthesis System (BLISS) [7], among others. As a result, research in comparative study becomes valuable in evaluating the capabilities and effectiveness of each proposed MDO method, as well as their limitations.

\author{
Ruben E. Perez, Hugh T. Liu \\ Institute for Aerospace Studies \\ University of Toronto \\ Toronto, ON, Canada, M3H 5 T6 \\ rperez@utias.utoronto.ca,liu@utias.utoronto.ca
}

A number of comparative studies have been reported over the past decade. On the one hand, the evaluation of MDO methods is still based on the efficiency of optimization algorithms. For example, Hulme and Bloebaum compare several MDO methods, including the MDF and IDF, with five analytical examples of varying size and complexity $\left.{ }^{8}\right]$. The evaluation is based on metrics such as the number of iteration cycles, design variables, and the accuracy. Chen et al. $\left[{ }^{9}\right]$ use the same metrics to evaluate three different MDO methods (CO, CSSO, and BLISS), with two application examples. On the other hand, the MDO methods distinguish themselves from normal optimization algorithms in that their architecture is an inseparable part of the problem formulation. Therefore, comparison of MDO methods must also address the different formulation structures. Kodiyalam, Alexandrov of NASA also pointed out the importance of formulation evaluation apart from the traditional optimization metrics. Their initial work in evaluation of the MDF, IDF, and CO methods suggest considerations of formulation oriented metrics, such as the generality, robustness, and performance $\left[{ }^{10},{ }^{11},{ }^{12}\right]$. However, details of these suggested metrics are not presented. Therefore, much work still needs to be done in evaluation of MDO methods, not only for its informative "systematic study", but also for its contribution in establishing standards or guidelines in MDO methods selection and testing.

In this paper, we present an extension of the comparative study of those presented in Refs. [9-12]. First of all, we extend the number of comparison subjects, to include all five aforementioned MDO methods (MDF, IDF, CO, CSSO, and BLISS). Secondly, we propose an extended set of metrics, taking into account both the formulation considerations and the optimization performance criteria. Quantitative details of the evaluation metrics are also presented. The investigation includes a similar analytical example to that presented in Ref. 6 for illustration. Furthermore, a supersonic business jet case is applied to demonstrate the evaluation of MDO methods in aircraft conceptual design. 


\section{MDO Methods Description}

The MDO problem consists of multiple interacting disciplines. Assume each discipline is described by the following mathematical representation:

$$
y_{i}=f\left(x_{i}, y_{j}, z\right), \quad i, j=1, \ldots, n \quad j \neq i
$$

where $n$ is the total number of coupled disciplines, counted by $\mathrm{i}$, representing the $\mathrm{i}^{\text {th }}$ discipline, $x_{\mathrm{i}}$ is the local variable vector, the vector $y_{\mathrm{j}}$ corresponds to interdisciplinary couplings, and $z$ denotes the global or shared variable vector. In addition, a set of parameters $p$ is required for each discipline but it does not vary over a design process. These parameters may or may not be shared by multiple disciplines.

\section{A. Multi-Disciplinary Feasible Design (MDF)}

The MDF has the simplest formulation for solving MDO problems $\left[3,{ }^{13}\right]$. Its formulation links a multidisciplinary design analysis (MDA) with an optimizer (Fig. 1) to find the optimal global $\mathrm{z}$ and local variables $\mathrm{x}$, for a given objective function and constraints. It reaches a multidisciplinary feasible state for an entire set of disciplines. In a MDA disciplinary state, variables $y$ are typically found by a Gauss-Seidel iteration between various disciplinary analyses, based on the given set of input parameters $x$ and $z$ and estimated coupling states. The MDF approach can be stated as:

$$
\begin{array}{ll}
\min _{z, x} & f\left(z, y_{i}\left(x, y_{j}, z\right), x\right) \quad i, j=1, \ldots, n \quad j \neq i \\
\text { s.t. } & g\left(z, y_{i}\left(x, y_{j}, z\right)\right) \leq 0
\end{array}
$$

where $f$ is the objective function and $g$ represent all the global and local system constraints.

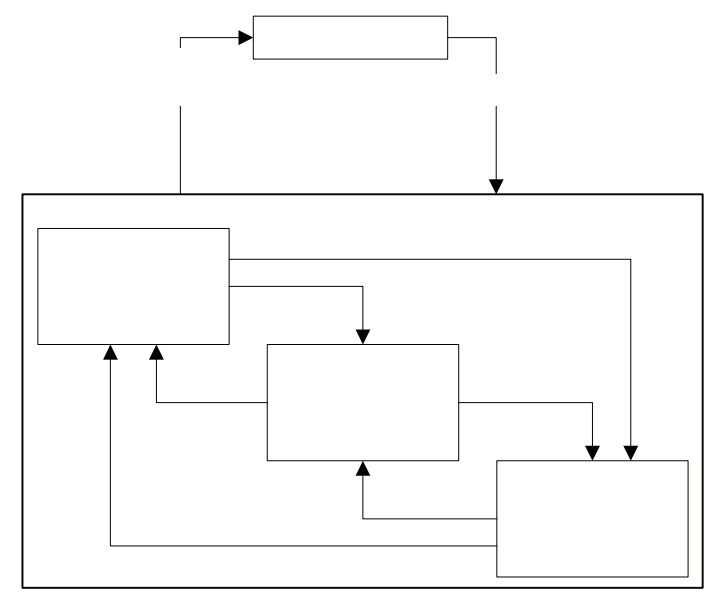

Figure 1. Multidisciplinary Feasible Method

\section{B. Individual Discipline Feasible (IDF)}

The IDF method provides an approach to avoid a complete MDA optimization. The method decouples the disciplinary analyses but keeps a unified optimization [4] (Fig. 2). It allows the optimizer to drive the individual disciplines to a multidisciplinary feasibility and optimality, by imposing feasibility constraints with extra coupling variables $y^{\prime}$ that are introduced in the formulation $\left[{ }^{14}{ }^{15}\right]$. The local disciplines can be feasible but the complete system may not be feasible until the optimization process converges. The IDF formulation can be stated as:

$$
\begin{array}{ll}
\min _{z, y^{\prime}, x} & f\left(z, y_{i}\left(x, y_{j}{ }^{\prime}, z\right), x\right) \quad i, j=1, \ldots, n \quad j \neq i \\
\text { s.t. } & g\left(z, y_{i}\left(x, y_{j}{ }^{\prime}, z\right), x\right) \leq 0 \\
& y_{i}{ }^{\prime}-y_{i}\left(x, y_{j}{ }^{\prime}, z\right)=0
\end{array}
$$

where $y^{\prime}$ is the extra coupling variable vector created to decouple the disciplinary analysis.

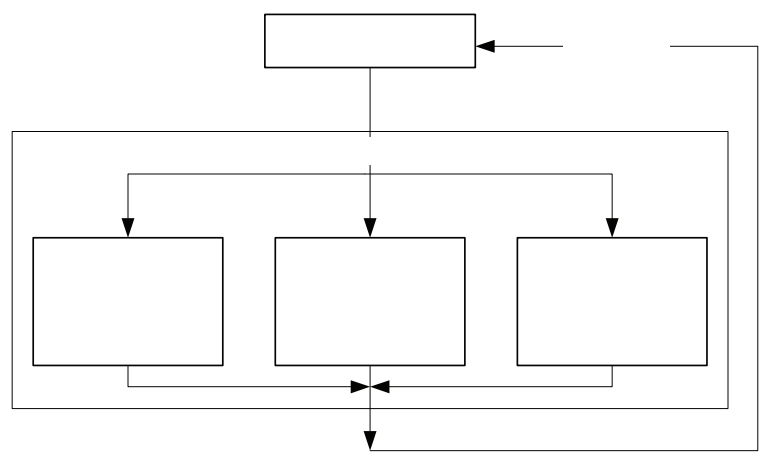

Figure 2. Individual Discipline Feasible Method 


\section{Collaborative Optimization (CO)}

Collaborative Optimization (CO) introduces a decomposed and decentralized bi-level optimization scheme [5] (Fig. 3). A system level optimization is responsible for providing target values for global design variables $z$ and system responses $y$. A local disciplinary level optimization assures that the discrepancies between disciplines vanish (to ensure multidisciplinary feasibility) by enforcing compatibility constraints. It is modelled to minimize the interdisciplinary discrepancies while satisfying specific local constraints. The $\mathrm{CO}$ formulation can be stated at the system level as:

$$
\begin{array}{ll}
\min _{z_{S L}, y_{S L}} & f\left(z_{S L}, y_{S L}\right) \\
\text { s.t. } & J_{i}\left(z_{S L}, z_{i}^{*}, y_{S L}, y_{i}^{*}\left(x_{i}^{*}, y_{j}, z_{i}^{*}\right)\right)=0 \quad \begin{array}{c}
i, j=1, \ldots, n \\
\end{array}
\end{array}
$$

$J$ represents the compatibility constraints, one for each discipline ( $n$ disciplines in total), and $z^{*}, y^{*}$ and $x^{*}$ are the optimal disciplinary optimization level results.

The $i^{\text {th }}$ disciplinary level optimization problem is formulated as:

$$
\begin{array}{ll}
\min _{z, y, x} & J_{i}=\sum\left(z_{S L}-z_{i}\right)^{2}+\sum\left(y_{S L}-y_{i}\right)^{2} \\
\text { s.t. } & g_{i}\left(x_{i}, z_{i}, y_{i}\left(x_{i}, y_{j}, z_{i}\right)\right) \leq 0
\end{array}
$$

where $g$ is the specific disciplinary constraint.

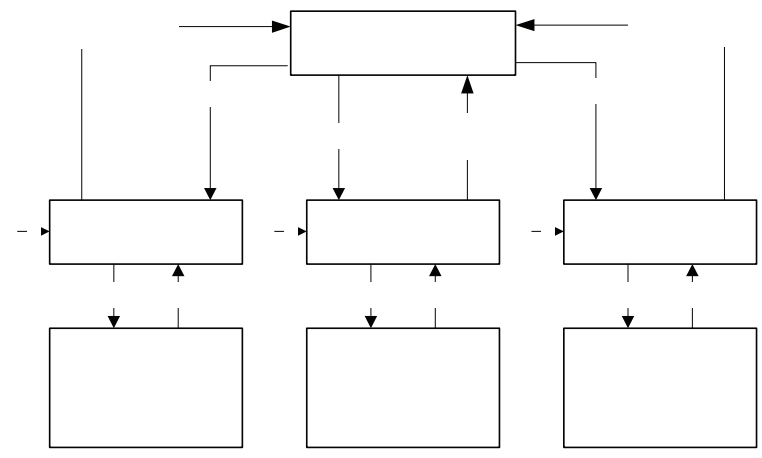

Figure 3. Collaborative Optimization Method

\section{Concurrent Subspace Optimization (CSSO)}

The Concurrent Subspace Optimization Method (CSSO) method is a decomposition-based strategy allowing concurrent optimization (Fig. 4). It takes advantage of the fact that the approximations of non-local disciplinary states help to understand the influences of local disciplinary variables on system level constraints and objective functions [6]. A specific performance is approximated in each disciplinary optimization to simulate other discipline state variables responses. Similarly, the system level optimization uses the approximation models to replace the required disciplinary analysis. Then the disciplinary level models are updated based on the optimized disciplinary states. The $i^{\text {th }}$ discipline optimization can be stated as:

$$
\begin{array}{ll}
\min _{z, y} & f\left(z, y\left(x_{i}, y_{j}^{a p p}, z_{i}\right), y_{j}^{a p p}\right) \quad \begin{array}{c}
i, j=1, \ldots, n \\
j \neq i
\end{array} \\
\text { s.t. } & g_{i}\left(x_{i}, z, y_{i}\left(x_{i}, y_{j}^{a p p}, z_{i}\right), y_{j}^{a p p}\right) \leq 0
\end{array}
$$

where $y_{\mathrm{j}}^{\text {app }}=y_{\mathrm{j}}^{\text {app }}\left(z, x_{j}\right)$ represents the other discipline approximate state responses. A complete multidisciplinary analysis is performed for each system level design to generate a multidisciplinary feasible design which is used to update the approximated system model. The system level optimization is stated as:

$$
\begin{array}{ll}
\min _{z, y^{a p p}} & f\left(z, y^{a p p}\right) \\
\text { s.t. } & g\left(z, y^{a p p}\right) \leq 0
\end{array}
$$

In addition, multidisciplinary analyses are performed with the local disciplinary level designs to further improve the models.

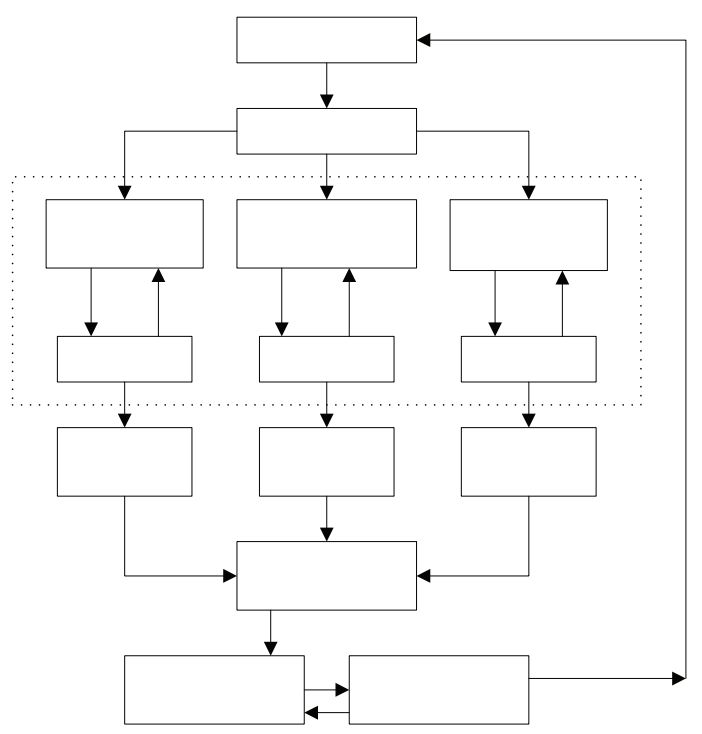

Figure 4. CSSO Method 


\section{E. Bi-Level Integrated System Synthesis Method}

The Bi-Level Integrated System Synthesis (BLISS) method (Fig. 5) is a decomposition extension of the global sensitivity equations (GSE) method $\left[7,{ }^{16}\right]$. It calculates the total derivative of the coupling values $y$ with respect to local sensitivities. Each discipline is optimized by varying their local variables $x$, while holding the global variables $z$ constant and minimizing the disciplinary objective under local constraints. The global variables are utilized by the system level optimization only. Total derivatives, obtained from GSE, are used to predict the effects of each set of variables on the objective function. The optimization of the ${ }_{i}^{\text {th }}$ discipline takes the form:

$$
\begin{array}{ll}
\min & d\left(f, x_{i}\right)^{T} \Delta x_{i} \\
\text { s.t. } & g_{i}\left(x_{i}\right) \leq 0
\end{array}
$$

where $d\left(f, x_{i}\right)^{T}$ is the local total derivative of the objective function with respect to the local variables and disciplines. It includes the indirect effects of these variables on other disciplines. The term $d\left(f, x_{i}\right)^{T} \Delta x_{i}$ corresponds to the first order predicted objective function change due to a change in $x_{i}$. The system level objective in the BLISS formulation is strongly related to the objective functions of the disciplines and it is expressed in terms of a first order Taylor series expansion:

$$
\begin{array}{ll}
\min & \Phi=d\left(y_{1, i}, x_{1}\right)^{T} \Delta x_{1}+d\left(y_{1, i}, x_{2}\right)^{T} \Delta x_{2}+\ldots \\
\text { s.t. } & g(z, y(x, z), x)
\end{array}
$$

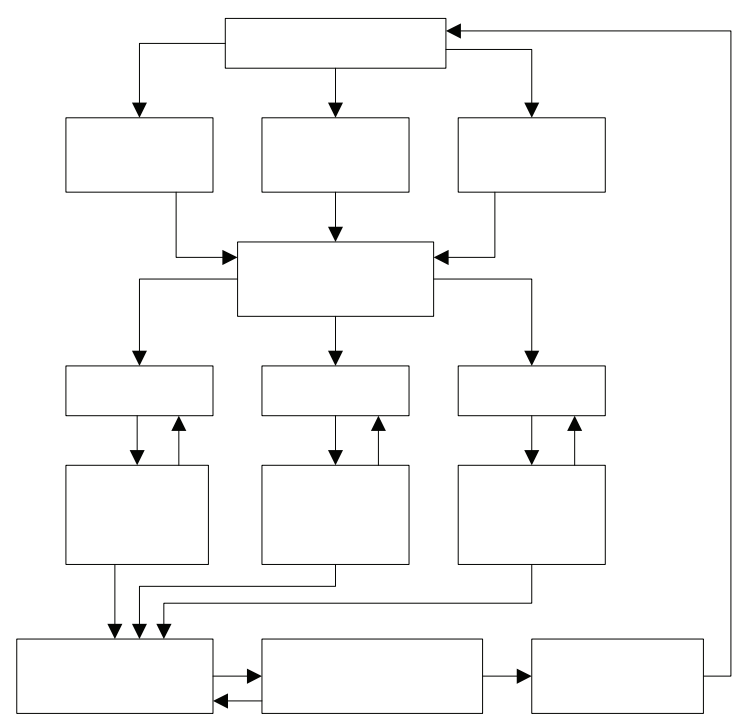

Figure 5. BLISS Method

\section{Methods Comparison}

\section{A. Metrics for Performance}

As we mentioned in Section I, a successful solution of the MDO problem not only depends on the efficient optimization algorithms, but also relies on effective architecture formulations. Therefore, it is necessary to take into account both of these considerations in any comparative or evaluation investigation. The optimization performance considerations are captured by traditional metrics such as the computational efficiency, accuracy, and so on. The architecture formulation consideration, however, has not been addressed extensively or quantitatively, to the knowledge of the authors. In this paper, we propose the following set of metrics to address both optimization and architectural considerations simultaneously for any MDO method.

- Simplicity - ease of implementation and modification. Simpler methods require less time to be modified and are easier to adapt to different problems. Simplicity is measured in terms of the total number of optimizers and optimizer variables required to implement a specific architecture in the scope of a given example.

- Transparency - the capacity to understand and extend the mathematical model from which the method is derived. For example, a probability-based method can be seamlessly integrated into a transparent formulation, which does not require major changes of the architecture to accomplish the integration.

- Portability - the feasibility to integrate a given method into the existing organizational structures. This metric takes into account the ability of a given architecture to take advantage of the division of labour and the autonomy of disciplinary specialist.

- Efficiency - the computational effort required to obtain an optimal multidisciplinary feasible design. In our study, the efficiency is measured based on the total number of disciplinary evaluations. It also includes the sensitivity analysis and the approximation analysis evaluations.

- Accuracy - the capacity of obtaining accurate optimal multidisciplinary feasible designs over the defined design space. In our study, the accuracy of a MDO method is determined by comparing the achieved results with given optimal values. 
Details of the quantitative descriptions of the metrics are presented in the following two case study examples.

\section{B. Test Case 1: Analytical Example}

The first example is selected from Ref. 6. It is described by the following equation:

$$
\begin{aligned}
& \min f=x_{2}^{2}+x_{3}+y_{1}+e^{-y_{2}} \\
& \text { s.t. } \quad\left[\begin{array}{l}
g_{1}=\left(\frac{y_{1}}{3.16}\right)-1 \geq 0 \\
g_{2}=1-\left(\frac{y_{2}}{24}\right) \geq 0 \\
-10 \leq x_{1} \leq 10 \\
0 \leq x_{2} \leq 10 \\
0 \leq x_{3} \leq 10
\end{array}\right. \\
& \text { where: }\left[\begin{array}{l}
y_{1}=x_{1}^{2}+x_{2}+x_{3}-0.2 y_{2} \\
y_{2}=\sqrt{y_{1}}+x_{1}+x_{3}
\end{array}\right.
\end{aligned}
$$

The same initial point $x=\{1,5,2\}, y=\{10,4\}$, is used for all analysis. The minimum is located at $x=$ $\{1.9776,0,0\}, y=\{3.7553,3.1834\}$.

\section{Test Case 2: Aircraft Conceptual Design}

A second example corresponds to the problem used by NASA to present the BLISS algorithm $\left[{ }^{17}\right]$, and provides a representative example of aircraft conceptual design. The goal is to maximize the range of a supersonic business jet subject to individual disciplinary constraints. Four coupled disciplinary systems are used (Fig. 6), representing structures, aerodynamic, propulsion, and performance. The first three disciplines are fully coupled since they share common variables and exchange computed states. The fourth discipline (performance) receives information from the others to evaluate the range performance of the design. Structures and weights are coupled to aerodynamic and propulsion. This is expected since aerodynamic loads cause changes in aircraft structural deflection that in turn changes the aerodynamics characteristics of the aircraft. Similarly, the propulsion and weights are coupled. The thrust required is dependent on the total aircraft weight, including the engine weight, which is also the function of thrust. A detailed description of this example is presented in Ref. [7].

\section{Results and Comparison}

The five MDO methods described in Section II are applied to the both test cases. The evaluation is based on the proposed metrics. Sequential Quadratic Programming (SQP) is used for all cases to maintain uniformity in the comparisons. A finite difference approach is used when sensitivity calculations are required. The reported number of function evaluations includes all optimization, sensitivity, and approximation callings.

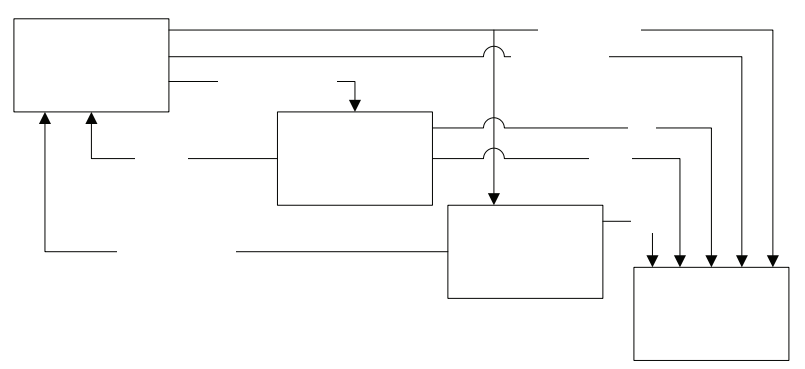

Figure 6. Supersonic Business Jet Example

\section{A. Simplicity}

Simplicity in MDO methods is sought to reduce the amount of implementation time and modification of the methodology to suit different optimization tasks. Quantitative criterion of the simplicity metric is chosen to be the number of optimizers required by the different MDO methods. Table 1 shows that the simplest method is the MFD approach since it is based on an integrated scheme, and requires only one optimizer. IDF presents an additional level of complexity due to the increase in the number of variables, used by the centralized optimizer. They are generated from the decoupling disciplinary outputs. CO presents an intermediate level of complexity due to its bi-level optimization scheme. Under a similar bi-level structure, the CSSO and BLISS methods also will require additional variables to support the approximation and the disciplinary sensitivity analyses respectively. They are deemed to share the high level of complexity. Simulation results of the two test cases are listed in Table 2 and Table 3 respectively. They demonstrate the same conclusions.

\section{B. Transparency}

Modelling transparency and simplicity are inherently correlated. In general, a transparent method is easy to understand and straightforward in implementation. From the modelling perspective, both MFD and IDF methods represent the same 
transparency where the mathematical model and optimization objectives can be easily formulated and modified. The transparency of $\mathrm{CO}$ formulation is similar to MFD and IDF, but it requires careful attention in the disciplinary objective definition due to its interdisciplinary compatibility formulation. While CSSO is similar to CO, the definition and treatment of approximations can be fairly difficult. It may become difficult in choosing proper approximation algorithms. BLISS is a less transparent approach since it requires a sensitivity analysis, in which formulation can be challenging for applications with large number of interacting parameters.

\begin{tabular}{|c|c|c|}
\hline $\begin{array}{l}\text { MDO } \\
\text { Method }\end{array}$ & No. Optimizers & Additional Variables \\
\hline MFD & 1 & - \\
\hline IDF & 1 & Couplings \\
\hline CSSO & $\mathrm{n}+1$ & $\begin{array}{c}\text { couplings \& } \\
\text { approximations }\end{array}$ \\
\hline $\mathrm{CO}$ & $\mathrm{n}+1$ & Couplings \\
\hline BLISS & $\mathrm{n}+1$ & $\begin{array}{l}\text { couplings and } \\
\text { sensitivities }\end{array}$ \\
\hline
\end{tabular}

\begin{tabular}{c|ccc}
\multicolumn{4}{c}{ Table 2. Analytical Example Optimizer Variables } \\
MDO & Coordination & Discipline 1 & Discipline 2 \\
Method & 3 & - & - \\
\hline MFD & 3 & - & - \\
IDF & 5 & 2 & 1 \\
CSSO & 3 & 4 & 3 \\
CO & 5 & 2 & 1 \\
BLISS & 3 & &
\end{tabular}

Table 3. Aircraft Design Example Optimizer Variables

\begin{tabular}{c|ccccc}
$\begin{array}{c}\text { MDO } \\
\text { Method }\end{array}$ & Coord & Structures & $\begin{array}{c}\text { Aero- } \\
\text { dynamics }\end{array}$ & Propulsion & Performance \\
\hline MFD & 10 & - & - & - & - \\
IDF & 20 & - & - & - & - \\
CSSO & 10 & 8 & 7 & 7 & - \\
CO & 16 & 8 & 10 & 4 & 6 \\
BLISS & 6 & 2 & 1 & 1 & -
\end{tabular}

\section{Portability}

The centralized optimization methods (MFD, IDF) allow for an integrated solution. However, they are not flexible in distributing the workload. Furthermore, they do not adapt well to the existing organizational structures, where different disciplines are often isolated in separate disciplinary sections. This is obvious in an aircraft design process. On the contrary, the CO, CSSO, and BLISS methods can be adapted directly to existing organizational structures, and can take advantage of distributed computing architectures. However, from the organizational perspective, CSSO is not efficient since each disciplinary group needs to make approximations of their own disciplinary state responses. Furthermore, a full system analysis is required each iteration. BLISS presents a balanced alternative method, especially in dealing with large number of coupling variables. It exploits the GSE approach in a disciplinary distributed environment. Unfortunately, the BLISS suffers from computational burden in calculating the sensitivities for each discipline. Often there exist no analytic sensitivity formulas, and numerical solutions are required. Besides, sensitivity analyses are not always available in practice.

\section{Computational Efficiency}

Table 4 shows the total number of evaluations used by each MDO method for the Test Case 1: the analytical example. IDF presents the best strategy with the lowest number of disciplinary evaluations. As expected, the bi-level decomposition based methods take a larger number of evaluations to find a feasible solution. Compared with $\mathrm{CO}$ and CSSO, BLISS shows the best performance in terms of the number of system evaluations as well as the disciplinary evaluations. This is due to the fact that sensitivity functions are analytic in this case. On the other hand, the CSSO method finds the solution much faster, since the approximations of the analytical equations are computationally inexpensive and the function is convex and continuous, leading to faster convergence.

Table 5 shows the number of evaluations for the aircraft design example. Note that a large number of function evaluations are required by the MFD method in this example. It shows the difficulty in using a centralized optimizer for complex MDO problems. The IDF shows some improvement as compared to MFD. As expected, decomposed approaches tend to be computationally expensive. Note that CO presents the average lowest number of disciplinary function evaluations, when compare to CSSO and BLISS. They require additional function evaluations to create valid approximations or to perform sensitivity analysis respectively. The low number of coordination iterations of BLISS indicates the performance advantage of having detailed sensitivity information between the design and couplings variables.

\section{E. Accuracy}

Results from all the five MDO methods of the analytical example are shown on Table 6. It should 
be noted that all MDO methods find the multidisciplinary feasible solution. The MFD and IDF methods provide the exact optimum. Also it can be seen that decoupling of variables provided by the IDF method does not affect the final multidisciplinary feasible point. Decomposition based methods CO, CSSO and BLISS find results close to the solution. Minor discrepancies exist in the solutions. They are originated from the interdisciplinary compatibility limits, and the degree of approximation imposed for $\mathrm{CO}$ and CSSO.

\begin{tabular}{c|ccc}
\multicolumn{2}{c}{ Table 4. Analytical Example Disciplines Evaluations } \\
MDO & $\begin{array}{c}\text { Coordination } \\
\text { Evaluations }\end{array}$ & $\begin{array}{c}\text { Discipline } 1 \\
\text { Evaluations }\end{array}$ & $\begin{array}{c}\text { Discipline 2 } \\
\text { Evaluations }\end{array}$ \\
Method & 24 & 216 & 216 \\
MFD & 24 & 54 & 54 \\
IDF & 62 & 528 & 528 \\
CSSO & 20 & 6106 & 4515 \\
CO & 249 & 95 & 95 \\
BLISS & 40 & &
\end{tabular}

Table 5. Aircraft Design Example Disciplines Evaluations

\begin{tabular}{l|lllll}
$\begin{array}{l}\text { MDO } \\
\text { Method }\end{array}$ & Coord. & Structures & $\begin{array}{l}\text { Aero- } \\
\text { dynamics }\end{array}$ & Propulsion & Performance \\
\hline MFD & 1216 & 5215 & 5215 & 5215 & 5215 \\
IDF & 525 & 525 & 525 & 525 & 525 \\
CSSO & 1020 & 9154 & 8115 & 6742 & 4185 \\
CO & 1956 & 8731 & 7842 & 6985 & 5211 \\
BLISS & 60 & 9423 & 8349 & 6647 & 3986
\end{tabular}

Table 6. Analytical Example Results

\begin{tabular}{c|cccccc} 
& $x_{1}$ & $x_{2}$ & $x_{3}$ & $y_{1}$ & $y_{2}$ & $f$ \\
\hline Initial & 1 & 5 & 2 & 10 & 4 & 10 \\
Value & 1.9776 & 0 & 0 & 3.16 & 3.7553 & 3.1834 \\
MFD & 1.9776 & 0 & 0 & 3.16 & 3.7553 & 3.1834 \\
IDF & 1.976 & 0 & 0 & 3.16 & 3.7675 & 3.1831 \\
CSSO & 1.9786 & 0 & 0 & 3.16 & 3.7556 & 3.1835 \\
CO & 1.9776 & 0 & 0 & 3.1504
\end{tabular}

Table 7 shows the results for the aircraft design example. The MFD performs a full system analysis and reaches a poor multidisciplinary feasible point with low range values. It demonstrates the difficulty of this method to find the optimum when the number of coupling and variables increases. The IDF results present a similar behaviour compared to MFD. The decomposed approaches are shown to be the better alternative in this case. While these methods tend to be more computationally expensive, they achieve better feasibility levels. All three methods in this category ( $\mathrm{CO}, \mathrm{CSSO}$, and BLISS) achieve ranges in the order of $3000 \mathrm{~nm}$. BLISS obtains the most feasible solution in terms of number of active constraints, but it also has the most computationally expensive procedure due to the sensitivity analysis involved. $\mathrm{CO}$ presents similar results as those in BLISS, but it requires more computational time to find a solution.

\section{Summary}

Based on for the proposed metrics to evaluate the five representative MDO methods, two test cases are analyzed to determine the characteristics of each method. A summary is shown in Table 8. MFD is the most accurate method since it performs full disciplinary system analysis. Unfortunately, its efficiency suffers with the increase in complexity. . Furthermore, it is difficult to integrate all given analysis in a common centralized platform. IDF can be a feasible alternative to MDF when portability analysis is not an issue. Bi-level optimization schemes prove to be computationally expensive but their accuracy is similar to centralized methods. Their main advantage lies in the portability for distributed analysis. Therefore they might become efficient when using parallel computing.

Table 7. Aircraft Design Example Results

\begin{tabular}{|c|c|c|c|c|c|c|c|c|c|c|}
\hline & $\lambda$ & $x$ & $C_{f}$ & $T$ & $t / c$ & $h$ & $M$ & $A R$ & $\Lambda$ & $S_{r e f}$ \\
\hline $\begin{array}{l}\text { Initial } \\
\text { Value }\end{array}$ & 0.25 & 1.0 & 1.0 & 0.5 & 0.05 & 45000 & 1.6 & 5.5 & 55 & 1000 \\
\hline MFD & 0.1 & 0.78 & 0.75 & 0.26 & 0.06 & 36089 & 1.4 & 2.5 & 70 & 1500 \\
\hline IDF & 0.4 & 1.14 & 1 & 0.13 & 0.06 & 45898 & 1.8 & 6.1 & 55 & 906 \\
\hline CSSO & 0.4 & 0.91 & 0.88 & 0.25 & 0.07 & 55426 & 1.6 & 3.5 & 52 & 1104 \\
\hline $\mathrm{CO}$ & 0.4 & 0.84 & 0.99 & 0.21 & 0.08 & 59154 & 1.7 & 3.6 & 45 & 1208 \\
\hline \multirow[t]{2}{*}{ BLISS } & 0.4 & 0.75 & 0.75 & 0.16 & 0.06 & 60000 & 1.4 & 2.5 & 70 & 1500 \\
\hline & $W_{t}$ & $W_{f}$ & $\Theta$ & $\mathbf{L}$ & D & L/D & SFC & We & ESF & Rang \\
\hline $\begin{array}{l}\text { Initial } \\
\text { Value }\end{array}$ & 41195 & 11254 & 1.02 & 46231 & 5264 & 9.5 & 0.88 & 6550 & 0.536 & 3378 \\
\hline MFD & 63532 & 19350 & 0.97 & 63532 & 19270 & 3.3 & 1.86 & 20021 & 1.5 & 517 \\
\hline IDF & 48789 & 21850 & 1.00 & 48789 & $967 \theta$ & 15 & 1.84 & 9997 & 1.00 & 1420 \\
\hline CSSO & 47891 & 19854 & 1.02 & 47841 & 7561 & 11 & 1.54 & 8461 & 0.84 & 3105 \\
\hline $\mathrm{CO}$ & 46828 & 16241 & 1.06 & 46828 & 5332 & 8.8 & 1.15 & 6739 & 0.53 & 3435 \\
\hline BLISS & 51411 & 7306 & 1.00 & 51411 & 13478 & 3.8 & 1.11 & 7058 & 0.55 & 3235 \\
\hline
\end{tabular}


Table 8. MDO Comparative Summary

Accuracy Efficiency Transparency Simplicity Portability

$\begin{array}{ccccc}\text { MFD } & \text { IDF } & \text { MFD } & \text { MFD } & \text { CO } \\ \text { IDF } & \text { BLISS } & \text { IDF } & \text { IDF } & \text { CSSO } \\ \text { BLISS } & \text { CSSO } & \text { CO } & \text { CO } & \text { BLISS } \\ \text { CO } & \text { CO } & \text { CSSO } & \text { CSSO } & \text { IDF } \\ \text { CSSO } & \text { MFD } & \text { BLISS } & \text { BLISS } & \text { MFD }\end{array}$

Aircraft conceptual design typically involves loosely coupled disciplines with a large number of global variables. Its goal is to find the optimum aircraft configurations. In the given example, $\mathrm{CO}$ proves to be the best choice of solution since its portability fits into existing organizational structures, and its simplicity makes it easy for modifications, a situation often encountered in conceptual design process. Furthermore, its transparency leads to easier extension for robust or probabilistic effects analyses. CSSO is efficient only for analytical formulations or for small-scale number of disciplines, since the system approximations increases the complexity of the implementation. However, approximations could prove beneficial to reduced computational burden, especially with expensive analyses and large number of couplings. BLISS provides certain amount of portability and seems to be more suitable in the preliminary and detailed aircraft design phases where highly coupled systems analysis is available.

\section{Conclusions}

This paper presents an extended evaluation of MDO methods. A simple analytical example and a more complex aircraft conceptual design example are both applied to evaluate the five MDO methods. The evaluation is based on our proposed metrics, which take into account formulation and the algorithm considerations. The quantitative description of the metrics provides a systematic approach in evaluating the MDO methods. Simulation results demonstrate the effectiveness of the proposed metrics, and concur with the experience from practice. Much work still needs to be done, not only for its informative "systematic study", but also for its contribution to establishing standards or guidelines in MDO selection and testing. Work under investigation will include additional examples, involving variance in the formulation complexity and the number of coupling and global variables. $\left.{ }^{1}\right]$ Current State of the Art in Multidisciplinary Design Optimization, MDO Technical Committee, White Paper, AIAA, Reston, VA, January 1991

$\left.{ }^{2}\right]$ Sobieszczanski-Sobieski J., "Multidisciplinary Design Optimization: An Emerging New Engineering Discipline", Proceeding of the World Congress of Optimal of Structural Systems, Rio de Janeiro, Brazil, August 1993

$\left.{ }^{3}\right]$ Cramer, "Problem Formulation for Multidisciplinary Optimization", SIAM Journal of Optimization, No.4, pp. 754-776, November 1994

[ $\left.{ }^{4}\right]$ Dennis, J.E., and Lewis, R.M., "Problem Formulations and Other Optimization Issues in Multidisciplinary Optimization," AIAA Paper 94-2196, AIAA Symposium on Fluid Dynamics, Colorado Springs, CO, June 1994

$\left.{ }^{5}\right]$ Braun R., Gage P., Kroo I., and Sobieszczanski-Sobieski J., Implementation and Performance Issues in Collaborative Optimization, Proceedings 5th AIAA/USAF MDO symposium, AIAA Paper 96-4017, Bellevue, WA, Sept. 1996

$\left.{ }^{6}{ }^{6}\right]$ Sell R.S., Batill S.M., and Renaud J.E., Response Surface Based Concurrent Subspace Optimization for Multidisciplinary System Design, American Institute of Aeronautics and Astronautics, AIAA Paper 96-0714

$\left.{ }^{7}\right]$ Sobieszczanski-Sobieski J., Jeremy S. A. and Robert R. S. Jr., Bi-Level Integrated System Synthesis (BLISS), National Aeronautics and Space Administration Technical Manual NASA/TM-1998-208715, August 1998

$\left[{ }^{8}\right]$ Hulme K.F., and Bloebaum C.L., A Comparison of Solution Strategies for Simulation-based Multidisciplinary Design Optimization, Proceedings Seventh AIAA/NASA/ISSMO Symposium on Multidisciplinary Analysis and Optimization, AIAA Paper 98-4977, St. Louis, MO, September, 1998, pp. 2143-2153

[9] Chen S., Zhang F., Khalid M., Evaluation of Three Decomposition MDO Algorithms, Proceedings of 23rd International Congress of Aerospace Sciences, Toronto, Canada, September, 2002

$\left[{ }^{10}\right]$ Alexandrov N.M., and Kodiyalam S., "Initial Results of an MDO Method Evaluation Study", Proceedings of the Seventh AIAA/USAF/NASA/ISSMO Symposium on Multidisciplinary Analysis and Optimization, St. Louis, MO, September 1998, pp. 1315-1327

$\left.{ }^{11}\right]$ Kodiyalam S., Evaluation of Methods for Multidisciplinary Design Optimization (MDO), Phase I, NASA Contractor Report, NASA CR-1998-208716, September 1998

$\left.{ }^{[2}\right]$ Kodiyalam S., and Yuan S., Evaluation of Methods for Multidisciplinary Design Optimization (MDO), Part II, NASA Contractor Report , NASA CR-2000-210313, November 2000

$\left.{ }^{13}\right]$ Cramer, "On Alternative problem Formulations for MDO", Proceedings Fourth AIAA/NASA/ISSMO Symposium on Multidisciplinary Analysis and Optimization, Cleveland, OH, Sept 1992, pp. 518-530

$\left[{ }^{14}\right]$ Haftka, R.T., Sobieszczanski-Sobieski J., and Padula S.L., "On Options for Interdisciplinary Analysis and Design Optimization", Structural Optimization, Vol. 4, No. 2, June 1992, pp. 65-74

$\left[{ }^{15}\right]$ Balling and Sobieszczanski-Sobieski J., "Optimization of Coupled Systems: A Critical Overview of Approaches",

\section{References}


American Institute of Aeronautics and Astronautics, AIAA Paper 94-4330-CP, Sept. 1994, pp. 753-773

$\left[{ }^{16}\right]$ Sobieszczanski-Sobieski J., "The Sensitivity of Complex, internally Coupled Systems", AIAA Journal, Vol. 28, No. 1, 1990, pp. 153-160

$\left.{ }^{17}\right]$ Sobieszczanski-Sobieski J., Agte J.S., and Sandusky R.R., Bi-Level Integrated System Synthesis (BLISS), NASA

Technical Manual, NASA TM-1998-208715 